\title{
Mental Disorders after Laryngectomy
}

\author{
Primoz Strojan Matjaz Zwitter \\ Institute of Oncology, Ljubljana, Slovenia
}

Laryngectomy is among the most mutilating treatments of cancer. The procedure brings physical incapacity and social stigmatization to patients who are commonly recruited from marginal social groups. Alcohol abuse, deficient diet, smoking and low social class - the most common risk factors for cancer of the head and neck - lead to poor social adaptation and to lower coping ability. We can therefore easily anticipate a high prevalence of psychological and psychiatric problems after laryngectomy. What comes as a surprise is not that psychiatric problems after laryngectomy are reported, but rather how little research has been done for a group of patients who need not only to be cured of cancer but also require more help and understanding.

Data on the incidence and survival can not reflect all the burden and complexity of a particular cancer. Psychosocial wellbeing is also an integral part of the assessment of quality of life and late consequences of treatment in patients cured of cancer. In head and neck oncology, this particular aspect of cancer research is much less investigated than for example in pediatric oncology or in breast cancer. In this respect, the article of Singer et al. on comorbid mental disorders in laryngectomees in this issue of ONKOLOGIE represents an important contribution to a neglected topic [1]. The authors are to be congratulated for their systematic approach to psychological and psychiatric problems after laryngectomy. Standardized psychological tests were used as assessment instruments, allowing a reliable quantitative comparison with the results of other similar studies. As the largest study on mental disorders in laryngectomees reported so far, this publication will be a reference for all further research in this area. In this brief comment, we would like to focus on reasons for scarce research on quality of life of patients after laryngectomy, on some findings of the current study, and on directions for further research.

We can only speculate on the reasons why this particular area of research has been neglected. Head and neck cancer is commonly considered a self-inflicted disease. In contrast to many other fields of oncology, very few patient support groups or associations of patients with head and neck cancer have been established. Male predominance, speech difficulties, and social origin contribute to the prevailing pattern of a 'lonely fighter'. The population of patients from the Singer study with its clinical and sociodemographic profile confirms these assumptions.

While the study by Singer et al. is extremely valuable as a reference for further work in this field, we feel that the proportion of patients with mental disorders after laryngectomy is grossly underestimated. The study reported only $8 \%$ of patients with alcohol dependency, $7 \%$ of those with major depression, and $5 \%$ with minor depression. These rather low figures may result from the methodology of the survey. First and above all, the mean interval between surgery and the interview was 6 years. Hence, only a minority of patients in the study were interviewed during the first few years after surgery when greatest psychological problems arising from mutilating surgery may be anticipated. We have very scarce data on coping with the consequences of laryngectomy during the most difficult first few years. One may speculate that some patients with very poor adaptation drowned into (or continued with) self-destructive behavior and did not survive long enough to be enrolled in this study.

Furthermore, the bias arises also from non-responders who may have refused participation in the interview because they were unwilling to report their behavior. Also, in a mail or phone interview, one may expect under-reporting of the drinking habits and of psychological or psychiatric disorders. Regarding alcohol abuse, longitudinal data - rather than a figure on a single point in time - would contribute to our understanding. What is the proportion of those who drank alcohol both before and after the disease; how many patients drank

\begin{tabular}{ll}
\hline KARGER & @ 2005 S. Karger GmbH, Freiburg \\
Fax +497614520714 & Accessible online at: \\
$\begin{array}{l}\text { E-mail Information@Karger.de } \\
\text { www.karger.com }\end{array}$ & www.karger.com/onk
\end{tabular}

Primoz Strojan, MD

Department of Radiotherapy,

Institute of Oncology

Zaloska 2, 1000 Ljubljana, Slovenia

Tel. +3861522 5253, Fax + 3865879400

E-mail pstrojan@onko-i.si 
before and stopped drinking after a serious disease; and how many started drinking in an attempt to escape the burden of the mutilating procedure? In addition, because of self-destructive behavior and lower-class social milieu of these patients, an assessment of the contribution of the diagnosis of cancer and the consequences of therapy to the incidence of mental disorders in this population is difficult and asks for specifically designed studies [2].

As the perception of morbidity is changing over time and between age groups, the analysis by treatment period and age of patients would certainly help to clarify the problem. Unfortunately, data on the site and extent of disease in the larynx at diagnosis are also missing. Comparison on the incidence of mental disorders with regard to marital status, socioeconomic status and level of education, occupational status and mode of speech would also be of interest. These data reflect subtle characteristics of the population under study and might be useful for interpretation of the results.

Only a longitudinal study could reveal the value of psychotherapy to alleviate the burden of laryngectomy. In this study, psychotherapeutic treatment was utilized in $11 \%$ of patients only. Unfortunately, we have no data on timing and on the results of such interventions. The other problem, as already stressed by Singer et al., lies in the medical care system itself: are the medical doctors familiar with psychodiagnostics; are the psychotherapists skilled in treating laryngectomees? In the eyes of many medical professionals and also of the lay public, attention to the psychological well-being of these patients may be unproductive. Thus, the question of the benefit of more intensive psychotherapy for patients with laryngeal cancer - at diagnosis and after laryngectomy - is to be defined.
We presume that further research will go in two directions. One is to evaluate pre-treatment versus post-treatment prevalence of mental disorders. The etiology of the cancer of the larynx is closely linked to cigarette smoking and to alcohol abuse which is, in turn, common denominator of mental distress and of psychiatric morbidity. Thus, the contribution of alcoholism as a competing cause of mental disorders should be assessed separately from the laryngectomy. The second direction of research is to compare the incidence of psychiatric morbidity between patients undergoing mutilating surgery and those treated with organ sparing protocols. The later are not without significant treatment-related late effects, but are nevertheless strongly preferred by patients $[3,4]$.

In conclusion, we would like to comment on the importance of this research for the choice of the treatment of laryngeal cancer. From many aspects, cancer of the larynx represents a unique challenge in oncology. When evaluating treatment results, the criterion 'functional outcome' is used more rigorously than usual. The devastating effect of total laryngectomy, and to a lesser degree of partial surgical techniques on voice, swallowing and respiration, compelled the clinicians to apply less mutilating, organ-sparing modalities [5-8]. No matter what approach will be accepted by the scientific community to win this challenge in the future, the question of psychosocial integration is equally relevant for all treatment options. Even more: the particular problem of comorbid mental disorders might serve as a tool to differentiate between more and less acceptable treatment strategies. At this point, we congratulate to Doctor Singer and her colleagues for their contribution to a neglected but crucial aspect of management of laryngeal cancer.

\section{References}

1 Singer S, Herrmann E, Welzel C, Klemm E, Heim M, Schwarz R: Comorbid mental disorders in laryngectomees. Onkologie 2005;28:631-638.

2 Nordgren M, Abendstein H, Jannert M, Boysen M, Ahlner-Elmqvist M, Silander E, Bjordal K, Hammerlid E: Health-related quality of life five years after diagnosis of laryngeal carcinoma. Int J Radiat Oncol Biol Phys 2003;56:1333-1343.

3 Richard JM, Sancho-Garnier H, Passey JJ, Luboinski B, Lefenvre JL, Dehesdin D, StromboniLuboinski M, Hill C: Randomized trial of induction chemotherapy in larynx carcinoma. Oral Oncol 1998;34:224-228.
4 Rudat V, Pfreundner L, Hoppe F, Dietz A: Approaches to preserve larynx function in locally advanced laryngeal and hypopharyngeal cancer. Onkologie 2004;27:368-375.

5 Andry G, Hamoir M, Leemans CR: The evolving role of surgery in the management of head and neck tumors. Curr Opin Oncol 2005;17:241-248.

6 6 The Department of Veterans Affairs Laryngeal Cancer Study Group: Induction chemotherapy plus radiation compared with surgery plus radiation in patients with advanced laryngeal cancer. N Engl J Med 1991;324:1685-1690.
7 Forastiere AA, Goepfert H, Maor M, Pajak TF, Weber R, Morrison W, Glisson B, Trotti A, Ridge JA, Chao C, Peters G, Lee DJ, Leaf A, Ensley J, Cooper J: Concurrent chemotherapy and radiotherapy for organ preservation in advanced laryngeal cancer. N Engl J Med 2003;349:2091-2098.

8 Budihna M, Soba E, Smid L, Zakotnik B, Strojan P, Cemazar M, Fajdiga I, Zargi M, Zupevc A, Lesnicar H: Inoperable oropharyngeal carcinoma treated with concomitant irradiation, mitomycin $\mathrm{C}$ and bleomycin - long term results. Neoplasma 2005;52:165-174. 